Ann. Biol. anim. Bioch. Biophys., 1977, 17 (6), 1107-1115.

\title{
Transfert et échange d'antìpyrine dans le lobule placentaire humain perfusé in vitro
}

\author{
par J. C. CHALLIER ( $\left.{ }^{(}\right)$, M. GUERRE-MILLO, M. O. RICHARD *, H. SCHNEIDER **, \\ M. PANIGEL, G. OLIVE * \\ Université Paris VI, Biologie de la Reproduction, Paris, France. \\ * CHU-Cochin, Phormacologie Biochimique, Poris, France. \\ ** Universifäts-Frauenklinik, Marburg, West-Germany.
}

Summary. Antipyrine transfer and exchange in the human placenta lobule during in vitro perfusion.

The purpose of this study was to determine whether substances were lost in the surrounding tissues during in vitro perfusion of human placental lobule. Studying transfer and exchange, we have compared the flow ratio computed from antipyrine extraction and transfer fractions to the flow ratio recorded with flowmeters. No significant difference was observed. Contrary to what would be supposed from the anatomy of maternal circulation, we concluded that, in steady state conditions, antipyrine loss was negligible with our perfusion system. The transport of antipyrine across the placenta was confirmed to be flow-dependent by the fact that the transfer and extraction fractions of that substance were related to flow ratio.

\section{Introduction.}

Une technique de perfusion in vitro du lobule placentaire humain a été mise au point par Panigel, Pascaud et Brun (1967). En utilisant cette technique, Schneider, Panigel et Dancis (1972) ont établi que chez l'homme, le transfert placentaire de l'antipyrine était limité par les débits de perfusion fotal et maternel. Par ailleurs, il a été démontré chez l'animal, que l'antipyrine traverse facilement la membrane placentaire. Elle n'est métabolisée que très lentement dans l'unité foto-placentaire (Meschia ef al., 1966). Elle n'est pas accumulée dans les tissus fotaux (Crenshaw ef al., 1968). A cause de ces propriétés, nous avons choisi d'étudier le transfert et l'échange de cette substance dans le lobule perfusé. En effet, si la technique de perfusion du lobule présente de nombreux avantages par rapport à la perfusion du placenta entier, le fait de perfuser uniquement une sous-unité isolée au sein du placenta peut présenter des inconvénients. Un inconvénient majeur serait l'existence de pertes de substance en

(1) Demande de tirés à part : J. C. Challier, Université Paris VI, Biologie de la Reproduction, 7, quai St-Bernard, 75230 Paris Cedex 05, France. 
direction des tissus adjacents, non perfusés. Pour évaluer ces pertes éventuelles, nous avons comparé la quantité d'antipyrine extraite d'un côté de la membrane placentaire à celle retrouvée de l'autre côté. Ceci a été fait au cours d'expériences de transfert de la mère au fœtus, sous l'action d'un gradient de concentration, d'une part. D'autre part, nous avons effectué des expériences d'échanges, sans gradient de concentration. Pour déterminer les pertes dans ce cas, nous avons comparé la quantité d'antipyrine transférée dans le sens materno-fœtal à celle transférée dans le sens fœio-maternel.

\section{Matériel et méthode.}

\section{Conditions de perfusion.}

Les circulations maternelle et fœtale de lobules choisis sur des placentas humains recueillis après la délivrance ou au cours de césariennes, sont perfusés suivant la technique décrite par Schneider ef al. (1972). Cette technique met en œuvre deux circuits de perfusion ouverts. L'un assure la perfusion de la circulation villositaire par les vaisseaux cathétérisés sur la plaque choriale, l'autre, la perfusion de l'espace intervilleux par deux canules en verre piquées dans la face déciduale. L'effluent maternel s'écoule librement par les orifices de la plaque basale. Pendant toute la durée de l'expérience, les débits et les pressions de perfusion sont contrôlés.

Au cours des deux types d'expériences, nos deux types de perfusat habituels, différents par leur capacité de transport de l'oxygène, sont utilisés :

a) Dans les expériences de transfert, la perfusion des circulations maternelle et fotale est faite avec du liquide de Earle, équilibré avec 95 p. 100 d'O $_{2}$ et 5 p. 100 de $\mathrm{CO}_{2}$. Le perfusat maternel contient de l'antipyrine froide $(10 \mathrm{mg} / 100 \mathrm{ml})$ ef de l'antipyrine marquée au ${ }^{14} \mathrm{C}$ (Amersham 20-50 m Ci/mM).

b) Dans les expériences d'échange, le perfusat consiste en une suspension d'hématies humaines dans du liquide de Earle, préparée selon la méthode décrite par Challier, Schneider ef Dancis (1976). Le perfusat esł équilibré avec un mélange d'air et de 5 p. 100 de $\mathrm{CO}_{2}$. Il contient de l'antipyrine froide $(10 \mathrm{mg} / 10 \mathrm{ml})$. On ajoute de plus, de l'antipyrine tritiée (N. E. N. $100-200 \mathrm{mCi} / \mathrm{mM}$ ) au perfusat fotal ef de l'antipyrine marquée au ${ }^{14} \mathrm{C}$ (N. E. N. $10-20 \mathrm{mCi} / \mathrm{mM}$ ) au perfusat maternel.

Les débits de perfusion sont mesurés sur des débitmètres Brooks R.2. 15. A, calibrés pour chaque perfusat. Du côté maternel, on mesure le débit artériel. Du côté fœetal, on mesure le débit artériel et le débit veineux, qui, dans nos conditions de perfusion sont égaux.

\section{Paramètres de transport et d'échange.}

Les paramètres employés pour décrire le passage transplacentaire de l'antipyrine sont :

- la fraction de transfert foetal :

$$
T_{F}=\left[\frac{C_{F v}}{C_{M a}}\right]_{14} \times 100 ;
$$


- la fraction de transfert maternel :

$$
\mathrm{T}_{\mathrm{M}}=\left[\frac{\mathrm{C}_{\mathrm{Mv}}}{\mathrm{C}_{\mathrm{Fa}}}\right]_{\mathrm{s}_{\mathrm{H}}} \times 100 ;
$$

- la fraction d'extraction maternelle :

$$
E_{M}=\left[\frac{C_{M a}-C_{M v}}{C_{M a}}\right]_{{ }_{14} \mathrm{C}} \times 100 .
$$

Avec : $C_{\mathrm{Fa}}$ : concentration d'antipyrine dans l'artère fœetale,

$\mathrm{C}_{\mathrm{Fv}}$ : concentration d'antipyrine dans la veine foetale,

$\mathrm{C}_{\mathrm{Ma}}$ : concentration d'antipyrine à l'entrée de l'espace intervilleux,

$C_{M v}$ : concentration d'antipyrine à la sortie de l'espace intervilleux.

Les indices ${ }^{3} \mathrm{H}$ et ${ }^{14} \mathrm{C}$ indiquent le marquage de l'antipyrine dont on suit le passage transplacentaire.

Lors des expériences d'échange, les clairances fœetale et maternelle sont déterminées en plus :

- la clairance maternelle :

$$
\left[\frac{C_{F v}}{C_{M a}}\right]_{{ }^{4} \mathrm{C}} \times Q_{F}
$$

- la clairance fotale :

$$
\left[\frac{C_{M v}}{C_{F a}}\right]_{s_{H}} \times Q_{M} .
$$

Avec les mêmes abréviations que ci-dessus, plus :

$$
\begin{aligned}
& Q_{F}: \text { le débit fœetal, } \\
& Q_{M}: \text { le débit maternel. }
\end{aligned}
$$

Dans chaque cas, les différentes concentrations sont mesurées lorsqu'un état stable est atteint dans la circulation fœtale et dans la circulation maternelle, c'est-àdire lorsque les concentrations restent constantes, en fonction du temps, dans les deux circulations.

III. Equations de transfert et d'échange.

1) Transfert.

Dans les expériences de transfert, il n'y a pas d'antipyrine dans le perfusat artériel fotal, donc $\mathrm{C}_{\mathrm{Fa}}=0$. Si la vitesse de disparition de l'antipyrine dans la circulation maternelle est égale à sa vitesse d'apparition dans la circulation fœale, il n'y a pas de perte de substance. On a l'équation suivante :

$$
\left[C_{M a}-C_{M v}\right]^{14} \mathrm{C} \times Q_{M}=\left[C_{F v}\right]^{14} \mathrm{C} \times Q_{F} .
$$

L'équation (6) est l'équation de conservation de l'antipyrine. Elle peut encore s'écrire :

$$
\left[\frac{C_{M a}-C_{M v}}{C_{F v}}\right]_{{ }_{14} \mathrm{~V}}=\frac{Q_{F}}{Q_{M}} .
$$


Le rapport $\mathrm{Q}_{\mathrm{F}} / \mathrm{Q}_{\mathrm{M}}$ est le rapport des débits mesurés sur les débitmètres. Le rapport $\left[\mathrm{C}_{\mathrm{Ma}}-\mathrm{C}_{\mathrm{Mv}} / \mathrm{C}_{\mathrm{Fv}}\right]^{\mathbf{a a}_{\mathrm{C}}}$ est égal au rapport de la fraction d'extraction maternelle $\left(E_{\mathrm{M}}\right)$ sur la fraction de transfert foetal $\left(T_{F}\right)$ de l'antipyrine marquée au ${ }^{14} \mathrm{C}$ ef sera considéré comme le rapport des débits calculé.

Le but de nos expériences, a été de démontrer l'égalité des deux termes de l'équation (7), c'est-à-dire, l'égalité des rapports des débits mesurés et des rapports des débits calculés. Si cette égalité est vérifiée, on considérera que les pertes d'antipyrine sont négligeables. Sinon, des pertes d'antipyrine se produisent, au cours de la perfusion du lobule isolé.

2) Echange.

Dans les expériences d'échanges, s'il n'y a pas de perte, la quantité d'antipyrine qui apparaît dans la circulation maternelle $-\left(C_{M v}-C_{M a}\right) \times Q_{M}$ - est égale à celle qui disparaît de la circulation fœitale $-\left(C_{F a}-C_{F v}\right) \times Q_{F}-$, pendant le même temps. Or, cette quantité d'antipyrine qui sort de la circulation foiale, est égale à celle qui y entre, pendant le même temps $-\left(C_{F v}-C_{F a}\right) \times Q_{F}-$ à condition que le flux net d'eau soit nul ef qu'il n'y ait pas de gradient de concentration en antipyrine entre les deux circulations. Dans nos expériences, nous n'avons pas de flux net d'eau puisque les débits artériel ef veineux fœtaux sont égaux. D'autre part, $\mathrm{C}_{\mathrm{Fa}}=\mathrm{C}_{\mathrm{Ma}}$. En effet, les concentrations d'antipyrine froide dans les deux perfusats sont égales et le gradient de concentration introduit par la différence d'activité spécifique du tritium et du carbone 14 est négligeable par rapport à la concentration de substance froide. Le flux net d'antipyrine est donc nul.

Dans ce cas, la vitesse d'apparition de l'antipyrine froide dans la circulation maternelle est égale à la vitesse d'apparition de l'antipyrine froide dans la circulation fœetale et l'on a l'équation :

$$
\left(\mathrm{C}_{\mathrm{Mv}}-\mathrm{C}_{\mathrm{Ma}}\right) \times \mathrm{Q}_{\mathrm{M}}=\left(\mathrm{C}_{\mathrm{Fv}}-\mathrm{C}_{\mathrm{Fa}}\right) \times \mathrm{Q}_{\mathrm{F}} .
$$

En divisant le premier membre de cette équation (8) par $C_{\mathrm{Fa}}$ et le second par $\mathrm{C}_{\mathrm{Ma}}$, on obtient :

$$
\frac{\mathrm{C}_{\mathrm{Mv}}-\mathrm{C}_{\mathrm{Ma}}}{\mathrm{C}_{\mathrm{Fa}}} \times \mathrm{Q}_{\mathrm{M}}=\frac{\mathrm{C}_{\mathrm{Fv}}-\mathrm{C}_{\mathrm{Fa}}}{\mathrm{C}_{\mathrm{Ma}}} \times \mathrm{Q}_{\mathrm{F}}
$$

a) Passage de la mère au foetus. $\mathrm{C}_{\mathrm{Fv}}-\mathrm{C}_{\mathrm{Fa}} / \mathrm{C}_{\mathrm{Ma}}$ est le rapport de la concentration d'antipyrine qui apparaît dans la circulation fotale sur la concentration maternelle artérielle. II représente le transfert de la mère au foetus $\left(T_{F}\right)$ que nous mesurons dans les expériences d'échange avec l'antipyrine marquée au carbone 14. D'où :

$$
\frac{C_{\mathrm{Fv}}-C_{\mathrm{Fa}}}{\mathrm{C}_{\mathrm{Ma}}}=\left[\frac{\mathrm{C}_{\mathrm{Fv}}}{C_{\mathrm{M}}}\right]_{{ }^{{ }^{4} \mathrm{C}}}
$$

b) Passage du fæetus à la mère. $\mathrm{C}_{\mathrm{Mv}}-\mathrm{C}_{\mathrm{Ma}} / \mathrm{C}_{\mathrm{Fa}}$ correspond à la concentration d'antipyrine qui apparaît dans la circulation maternelle par rapport à la concentration fœtale artérielle. C'est le transfert du fœetus à la mère $\left(T_{M}\right)$. On le mesure avec l'antipyrine tritiée. Donc:

$$
\frac{\mathrm{C}_{\mathrm{Mv}}-\mathrm{C}_{\mathrm{Ma}}}{\mathrm{C}_{\mathrm{Fa}}}=\left[\frac{\mathrm{C}_{\mathrm{Mv}}}{\mathrm{C}_{\mathrm{Fa}}}\right]_{{ }_{\mathrm{B}} \mathrm{H}} .
$$


En substituant ces rapports dans l'équation (9), on a :

$$
\left[\frac{C_{M v}}{C_{F a}}\right]_{{ }^{H} \mathrm{H}} \times Q_{M}=\left[\frac{C_{F v}}{C_{M a}}\right]_{{ }_{14} \mathrm{C}} \times Q_{F} .
$$

Ceci peut encore s'écrire :

$$
\frac{\left[\frac{C_{\mathrm{Mv}}}{\mathrm{C}_{\mathrm{Fa}}}\right]^{{ }^{3} \mathrm{H}}}{\left[\frac{\mathrm{C}_{\mathrm{Fv}}}{\mathrm{C}_{\mathrm{Ma}}}\right]_{{ }^{4} \mathrm{C}}}=\frac{\mathrm{Q}_{\mathrm{F}}}{\mathrm{Q}_{\mathrm{M}}} .
$$

Le rapport $\mathrm{Q}_{\mathrm{F}} / \mathrm{Q}_{\mathrm{M}}$ est le rapport des débits mesurés sur les débitmètres. Le premier terme de l'équation (11) est égal au rapport de la fraction de transfert maternel sur la fraction de transfert fœilal et sera considéré comme le rapport des débits calculé. Comme pour les expériences de transfert, la comparaison des deux termes de cette équation, nous permettra de déceler les pertes éventuelles d'antipyrine au cours de la perfusion.

\section{Analyses.}

L'antipyrine marquée au carbone 14 ou au tritium contenue dans les échantillons de perfusat a été extraite par le Chloroforme ( $1 v / 2 v, 3$ fois), après centrifugation. Les comptages de radioactivité ont été effectués sur un spectromètre à scintillation liquide (Packard ou Intertechnique). Le mélange scintillant utilisé est le Diotol ou l'Insta-Gel.

\section{TABLEAU 1}

\section{Expériences de transfert}

Les fractions de transfert et d'extraction sont données en pourcentage ; les débits en millilitres par minute. Les moyennes sont suivies de l'erreur type, sauf dans le cas des débits, lorsque ces derniers sont constants. Le test de Student pour échantillons appariés donne les résultats suivants : $\left(^{*}\right)$ différence signifi-

\begin{tabular}{|c|c|c|c|c|c|c|c|}
\hline Expériences & $\begin{array}{c}\text { Nombre } \\
\text { de } \\
\text { mesures }\end{array}$ & $\begin{array}{c}\text { Fraction } \\
\text { de transfert } \\
\text { fœetal } \\
\left(T_{F}\right)\end{array}$ & $\begin{array}{c}\text { Fraction } \\
\text { d'extraction } \\
\text { maternelle } \\
\left(E_{M}\right)\end{array}$ & $\begin{array}{c}\text { Rapport } \\
\text { des débits } \\
\text { calculé : } \\
\mathrm{E}_{\mathrm{M}} / \mathrm{T}_{\mathbf{F}}\end{array}$ & $\begin{array}{l}\text { Débit } \\
\text { fœetal } \\
\left(Q_{F}\right)\end{array}$ & $\begin{array}{c}\text { Débit } \\
\text { maternel } \\
\left(\mathrm{Q}_{\mathrm{M}}\right)\end{array}$ & $\begin{array}{c}\text { Rapport des } \\
\text { débits mesurés : } \\
\qquad \mathrm{Q}_{\mathrm{F}} / \mathrm{Q}_{\mathbf{M}}\end{array}$ \\
\hline $\begin{array}{l}\text { A III } \\
\text { A V } \\
\text { B I } \\
\text { B IV } \\
\text { B V } \\
\text { B VI } \\
\text { B VII }\end{array}$ & $\begin{array}{l}3 \\
3 \\
5 \\
4 \\
3 \\
5 \\
3\end{array}$ & $\begin{array}{l}64 \pm 1,4 \\
51 \pm 3,5 \\
49 \pm 1,7 \\
51 \pm 1,7 \\
44 \pm 2,0 \\
56 \pm 0,51 \\
26 \pm 1,9\end{array}$ & $\begin{array}{l}17 \pm 0,61 \\
9,3 \pm 1,9 \\
14 \pm 0,92 \\
20 \pm 1,6 \\
23 \pm 2,7 \\
19 \pm 0,86 \\
7,8 \pm 0,90\end{array}$ & $\begin{array}{l}0,25 \pm 0,015 \\
0,18 \pm 0,039 \\
0,29 \pm 0,027 \\
0,39 \pm 0,047 \\
0,51 \pm 0,040 \\
0,39 \pm 0,0040 \\
0,25 \pm 0,073\end{array}$ & $\begin{array}{c}6,0 \\
4,2 \pm 0,17 \\
5,8 \pm 0,12 \\
6,5 \\
5,0 \\
8,9 \pm 0,10 \\
5,0\end{array}$ & $\begin{array}{c}24 \\
23 \\
23 \pm 0,20 \\
16 \\
18 \\
23 \\
21\end{array}$ & $\begin{aligned} & 0,25 \\
0,18 \pm & 0,0067 \\
0,26 \pm & 0,0073 \\
& 0,40 \\
& 0,30 \\
0,39 \pm & 0,0040 \\
& 0,23\end{aligned}$ \\
\hline $\begin{array}{l}\text { Moyennes } \\
\text { générales }\end{array}$ & & $49 \pm 2,0\left(^{*}\right)$ & $16 \pm 1,1\left(^{*}\right)$ & $0,33 \pm 0,023\left({ }^{* *}\right)$ & & & $0,30 \pm 0,018(* *)$ \\
\hline
\end{tabular}
cative $(p<0,001),(* *)$ pas de différence au seuil de 5 p. 100. 


\section{Résultats.}

I. Transfert materno-fotal d'antipyrine.

Le tableau 1 présente les résultats de sept expériences de transfert. Pour des rapports de débits de perfusion compris entre 0,18 et 0,40 , les fractions de transfertfotal de l'antipyrine, en état stable, sont élevées : $49 \pm 2,0$ p. 100 , en moyenne. La fraction d'extraction maternelle moyenne est plus basse : $16 \pm 1,1 \mathrm{p}$. 100. La fraction de transfert fœial diffère significativement de la fraction d'extraction maternelle $(p<0,001)$.

Nous avons comparé les rapports des débits mesurés et les rapports des débits calculés. Le test de Student pour échantillons appariés, appliqué à ces deux séries de valeurs, montre qu'il n'y a pas de différence significative au seuil de 5 p. 100. On peut donc considérer que dans ce type d'expérience, les fuites d'antipyrine sont négligeables en état stable. Ces résultałs montrent que les fractions de transfert et d'extraction de l'antipyrine varient en fonction du rapport des débits de perfusion comme le laissait prévoir l'équation de conservation de l'antipyrine.

\section{Echanges fœto-maternel et materno-fœtal d'antipyrine.}

Le tableau 2 présente les résultats de quatre expériences d'échange. En état d'équilibre stable, les fractions de transfert maternel, mesurées à l'aide de l'antipyrine tritiée, diffèrent significativement $(P<0,001)$ des fractions de transfert fœelal, mesurées à l'aide de l'antipyrine marquée au carbone 14:36 2,6 p. 100 pour le passage de la mère au fœlus, contre $13 \pm 2,0$ p. 100 pour le passage du fœtus à la mère.

TABLEAU 2

Expériences d'échange

Les fractions de transfert sont données en pourcentage ; les débits en millilitres par minute. Les moyennes sont suivies de l'erreur type, sauf dans le cas des débits, lorsque ces derniers sont constants. Le test de Student pour échantillons appariés donne les résultats suivants : $\left(^{*}\right)$ différence significative $(p<0,001)$, (**) pas de différence au seuil de 5 p. 100.

\begin{tabular}{|c|c|c|c|c|c|c|c|}
\hline Expériences & $\begin{array}{c}\text { Nombre } \\
\text { de } \\
\text { mesures }\end{array}$ & $\begin{array}{c}\text { Fraction } \\
\text { de transfert } \\
\text { foetal } \\
\left(T_{F}\right)\end{array}$ & $\begin{array}{c}\text { Fraction } \\
\text { de transferf } \\
\text { maternel } \\
\left(T_{M}\right)\end{array}$ & $\begin{array}{l}\text { Rapport } \\
\text { des débits } \\
\text { calculé : } \\
T_{M} / T_{F}\end{array}$ & $\begin{array}{l}\text { Débit } \\
\text { fœtal } \\
\left(Q_{F}\right)\end{array}$ & $\begin{array}{c}\text { Débit } \\
\text { maternel } \\
\left(Q_{M}\right)\end{array}$ & $\begin{array}{l}\text { Rapport } \\
\text { des débits } \\
\text { mesurés : } \\
\mathrm{Q}_{\mathrm{F}} / \mathrm{Q}_{\mathrm{M}}\end{array}$ \\
\hline $\begin{array}{l}11-0 \\
12-0 \\
13-0 \\
14-0\end{array}$ & $\begin{array}{l}2 \\
3 \\
4 \\
4\end{array}$ & $\mid \begin{array}{l}37 \pm 7,6 \\
44 \pm 1,4 \\
38 \pm 4,8 \\
26 \pm 1,5\end{array}$ & $\begin{aligned} 7,8 & \pm 0,22 \\
10 & \pm 0,92 \\
23 & \pm 1,5 \\
8,1 & \pm 0,73\end{aligned}$ & $\begin{array}{l}0,22 \pm 0,039 \\
0,23 \pm 0,021 \\
0,64 \pm 0,085 \\
0,30 \pm 0,011\end{array}$ & $\left|\begin{array}{c}3,6 \\
2,1 \pm 0,23 \\
5,0 \pm 0,27 \\
2,8 \pm 0,075\end{array}\right|$ & $\begin{array}{c}16 \\
11 \pm 1,8 \\
8,1 \pm 0,71 \\
10 \pm 0,19\end{array}$ & $\begin{array}{c}0,23 \\
0,20 \pm 0,028 \\
0,63 \pm 0,032 \\
0,27 \pm 0,090\end{array}$ \\
\hline $\begin{array}{l}\text { Moyennes } \\
\text { générales }\end{array}$ & & $36 \pm 2,6\left({ }^{*}\right)$ & $13 \pm 2,0\left(^{*}\right)$ & $0,38 \pm 0,058(* *)$ & & & $0,36 \pm 0,054(* *)$ \\
\hline
\end{tabular}


Comme dans les expériences de transfert, le test de Student pour échantillons appariés a été appliqué aux deux séries de valeurs : rapport des débits mesurés et rapporł des débits calculés. Il n'y a pas de différence significative au seuil de 5 p. 100 . Dans ce type de perfusion, les fuites d'antipyrine sont également négligeables. Le même test statistique a été appliqué aux clairances fœetales et maternelles, calculées pour chaque expérience. Il n'existe pas de différence significative au seuil de 5 p. 100 entre les clairances fœtales et maternelles. En moyenne, la clairance maternelle est égale à 1,2 $\pm 0,17 \mathrm{ml} / \mathrm{mn}$, et la clairance fœtale 1,3 $\pm 0,14 \mathrm{ml} / \mathrm{mn}$.

\section{Discussion.}

Le placenta humain est composé d'unités morphologiques distinctes, les lobules, visibles sur la face déciduale. La partie foetale des lobules comprend en moyenne trois cotylédons, formés de villosités libres, irriguées par des ramifications des vaisseaux ombilicaux.

Le système vasculaire d'un cotylédon fœtal est limité sur tout son trajet par un endothélium. Lorsque les ramifications artérielles se rendant à des cotylédons voisins ont été ligaturées, la circulation du cotylédon perfusé est indépendante.

Du côté maternel le réseau vasculaire est dépourvu d'endothélium, ce qui est caractéristique d'un placenta hémochorial. Le sang maternel est en contact direct avec les villosités fœtales dans l'espace intervilleux. Des passages de sang maternel d'un lobule à l'autre sont possibles, car l'espace intervilleux est incomplètement cloisonné par des septa.

Selon Spanner (1935) le sang veineux maternel s'écoulerait par les ouvertures subsistant entre la plaque choriale et les septa, en direction du sinus marginal. De là, le sang regagnerait la circulation utérine. Cependant, les travaux de Ramsey ef al. (1966), in vivo, et de Panigel (1968) en perfusion in vifro, ont montré que des substances radio-opaques, arrivant au lobule par voie artérielle, restaient confinées dans l'espace intervilleux, le retour du sang veineux s'effectuant par les orifices de la plaque basale du lobule perfusé.

Les résultats que nous avons obtenus en suivant les passages d'antipyrine à travers le placenta, confirment qu'en état stable, il n'y a qu'une perte négligeable de substance au cours de la perfusion du lobule isolé. En effet, dans les expériences de transport, une perte de substances vers les tissus adjacents non perfusés devrait se traduire par une fraction d'extraction maternelle supérieure à celle correspondant au transfert. Dans un tel cas, on devrait trouver un écart entre le rapport des débits calculés et le rapport des débits mesurés. Or, nous ne trouvons pas, statistiquement, de différences entre ces deux valeurs. Dans les expériences d'échange également, une fuite de substance sur la circulation veineuse maternelle devrait se traduire par une fraction de transfert maternel diminuée et une fraction de transfert fœetal inchangée. Comme précédemment, une différence entre le rapport des débits calculé et le rapport des débits mesurés devrait être décelée, ce qui n'est pas le cas.

L'égalité des clairances fœtale et maternelle dans les expériences d'échange, indique que toute la quantité d'antipyrine extraite d'une circulation pendant un temps donné, se retrouve dans le même temps, de l'autre côté de la barrière placentaire, lorsqu'un état stable est atteint. 
Des résultats comparables ont été trouvés chez la chèvre (Rankin et Peterson, 1969). En perfusant le placenta entier in situ, ces auteurs montrent qu'il existe une égalité entre la fraction d'extraction maternelle du protoxyde d'azote, gaz dissous dans la circulation maternelle, et la fraction de transfert maternel de l'acétylène, gaz dissous dans la circulation fotale. Chez le lapin, Faber et Hart (1966) mesurent des débits apparents qui sont assimilables aux clairances. Ils étudient l'oxygène transporté dans la circulation fœtale et l'acétylène extrait de cette même circulation. En tenant compte de la consommation en oxygène du placenta, ils montrent que ces deux débits apparents sont égaux.

Ces travaux prouvent qu'une conservation des substances non métabolisables existe dans les systèmes anatomiquement clos comme les cotylédons du placenta de chèvre et le placenta de lapin. D'après nos résultats, il semble que ceci s'applique également au lobule placentaire humain. Or, le lobule, d'une part, n'est qu'une sousunité isolé au sein du placenta et, d'autre part, ne constitue pas un système entièrement clos, puisque l'espace intervilleux est continu. On peut se demander si un tel phénomène se produit in vivo. Le lobule que nous perfusons est entouré par un espace intervilleux maternel rempli de sang, mais sans courant sanguin. Du fait du fonctionnement intermittent des artères utéro-placentaires (Martin ef al., 1964), la même situation existe in vivo, au moins à certains moments, quand le tissu entourant le lobule perfusé n'est pas irrigué.

Nos résultats ont été obtenus en utilisant l'antipyrine. II est peu probable qu'ils découlent d'un artefact dû au choix de cette substance, puisqu'elle ne se lie pas aux protéines, qu'elle n'est pas métabolisée dans le placenta et qu'elle n'est pas accumulée dans les tissus placentaires, comme cela a été vérifié par Meschia et al. (1966) et Crenshaw et al. (1968). Nos résultats peuvent s'expliquer en supposant qu'en raison de la diffusibilité élevée de l'antipyrine, une équilibration rapide des concentrations s'établit entre le perfusat et les tissus des cotylédons voisins jusqu'à ce qu'un état stable soit atteint, durant lequel les pertes de substance dans l'espace intervilleux deviennent négligeables.

Nous avons montré également, que la fraction de transfert foetal et la fraction d'extraction maternelle de l'antipyrine étaient liées par le rapport des débits de perfusion, ce qui confirme le caractère débit-dépendant du passage transplacentaire de cette substance, démontré par Schneider et al. (1972). Ainsi, l'utilisation de l'antipyrine au cours de la perfusion in vitro du lobule isolé, permet de calculer le rapport des débits réel, et de tenir compte de variations d'une expérience à l'autre. En particulier, connaissant son transfert, on peut vérifier la bonne superposition des circulations fœtale et maternelle. Il reste à montrer que le transfert de l'antipyrine est maximum à un rapport de débit donné, pour que cette substance ait toutes les caractéristiques d'une substance de référence. Nous avons effectué cełte éłude en comparant le transfert de l'antipyrine à celui de l'eau tritiée (Challier et al., 1977).

\section{Conclusion.}

L'étude du passage de l'antipyrine de la mère au fœtus et du fœtus à la mère a montré que le lobule placentaire, perfusé in vitro, se comporte comme une sous-unité 
physiologiquement indépendante. Ce résultat est une justification supplémentaire de l'utilisation de lobules isolés pour l'étude des échanges placentaires, chez l'homme.

Reçu en mars 1977.

Accepté en septembre 1977.

\section{Références}

CHALLIER J. C., SCHNEIDER H., DANCIS J., 1976. In vitro perfusion of human placenta. V. Oxygen consumption. Amer. J. Obsiet. Gynec., 126, 261-265.

CHALLIER J. C., GUERRE-MILLO M., NANDAKUMARAN M., OliVE G., PANIGEL M., 1977. Placental transfer in vitro of water, antipyrine and inulin. Proc. Int. Union of Physiol. Sci., vol. 13, 129.

CRENSHAW C., HUCKABEe W. E., CURET L. B., MANN L., BARRON D. H., 1968. A method for the estimation of the umbilical blood flow in unstressed sheep and goat with some result of its application, Quart. J. exp. Physiol., 53, 65-75.

FABER J. J., HART F. M., 1966. The rabbit placenta as an organ of diffusional exchange. Comparison with other species by dimensional analysis. Circ. Res., 19, 816-833.

MARTIN C. B., MC GAUGHEY H. S., KAISER I. H., DONNER M. W., RAMSEY E. M., 1964. Intermittent functionning of uteroplacental arteries. Amer. J. Obstet. Gynec., 90, 819-823.

MESCHIA G., COTTER J. R., MAKOWSKY E. L., BARRON D. H., 1966. Simultaneous measurement of uterine and umbilical blood flows and oxygen uptakes. Quart. J. exper. Physiol., 52, 1-18.

PANIGEL M., PASCAUD M., BRUN J. L., 1967. Une nouvelle technique de perfusion de l'espace intervilleux dans le placenta humain isolé. Pathol. Biol., 15, 821.

PANIGEL M., 1968. Placental perfusion 15-25. In : WYNN R. M. Fetal homeosfasis. Appleton Century Crofts, Meredith Corp., New York.

RANKIN J. H. G., PETERSON E. N., 1969. Application of the theory of heat exchangers to a physiological study of the goat placenta. Circ. Res., 24, 235-250.

RAMSEY E. M., MARTIN C. B., McGAUGHEY H. S., KAYSER H. I., DONNER M. W., 1966. Venous drainage of the placenta in rhesus monkeys : radiographic studies. Amer. J. Obstet. Gynec., 95, 948-955.

SCHNEIDER H., PANIGEL M., DANCIS J., 1972. Transfer across the perfused human placenta of antipyrine, sodium and leucine. Amer. J. Obstet. Gynec., 114, 822-828.

SPANNER R., 1935. Mütterlicher und kindlicher Kreislauf der menschlichen Plazenta und seine Strombahnen. Z. Anat. Ent. Gesch., 105, 163-242. 